\title{
Boundary Value Problems For Caputo-Hadamard Fractional Differential Equations
}

\author{
Wafaa Benhamidaa ${ }^{a}$ Samira Hamani ${ }^{a}$, Johnny Henderson ${ }^{b}$ \\ a Laboratoire des Mathematiques Appliqués et Pures, Université de Mostaganem, Mostaganem, Algerie. \\ ${ }^{b}$ Department of Mathematics, Baylor University, Waco, Texas 76798-7328, USA.
}

\begin{abstract}
In this paper, we investigate the existence of solutions of a boundary value problem for Caputo-Hadamard fractional differential equations. Our analysis relies on classical fixed point theorems. Examples are given to illustrate our theoretical results.

Keywords: Fractional differential equation; Hadamard fractional derivative; Caputo-Hadamard fractional derivative; Hadamard fractional integral; fixed point.

2010 MSC: 26A33, 34A60
\end{abstract}

\section{Introduction}

The theory of fractional differential equations has received much attention over the past years and has become an important field of investigation due to its extensive applications in numerous branches of physics, chemistry, aerodynamics, electrodynamics of a complex medium, polymer rheology, etc. Fractional differential equations also serve as an excellent tool for the description of hereditary properties of various materials. As a consequence, the subject of fractional differential equations is gaining much importance and attention. For details, see [1, 4, 3, 5, 12, 17, 20, 28], and the references therein.

There has been a significant development in fractional differential equations in recent years; see the monographs of Kilbas et al. [22, Miller and Ross [25] and Samko et al. [27] and the papers of Delbosco and Rodino [13], Diethelm et al. [14], [15], [16], El-Sayed [17], Kilbas and Marzan [21], Mainardi [24] and Podlubny et al. [26].

Email addresses: benhamida.wafaa@yahoo.fr (Wafaa Benhamida), hamani_samira@yahoo.fr (Samira Hamani), johnny_henderson@baylor.edu (Johnny Henderson) 
There are several definitions of fractional derivatives, such as the definitions of Riemann-Liouville (1832), Riemann(1849), Caputo(1997), Grunwald-Letnikov(1867) and Hadamard(1891,[19]). The Caputo-Hadamard derivative is a new approach obtained from the Hadamard derivative by changing the order of its differential and integral parts. Despite the different requirements on the function itself, the main difference between the Caputo-Hadamard fractional derivative and the Hadamard fractional derivative is that the CaputoHadamard derivative of a constant is zero [20]. And the most important advantage of Caputo-Hadamard derivative is that it provided a new definition through which the integer order initial conditions can be defined for fractional.

Benchohra, Hamani and Ntouyas [5] studied the boundary value problem,

$$
\begin{gathered}
{ }^{c} D^{\alpha} y(t)=f(t, y(t)), \text { for a.e. } t \in[0, T], \quad 0<\alpha \leq 1, \\
a y(0)+b y(T)=c,
\end{gathered}
$$

where ${ }^{c} D^{\alpha}$ is the Caputo fractional derivative, $f:[0, T] \times \mathbb{R} \rightarrow \mathbb{R}$ is a given function, and $a, b$ and $c$ are real constants such that $a+b \neq 0$.

Motivated by the work above, in this paper, we concentrate on the following boundary value problem,

$$
\begin{gathered}
{ }_{H}^{c} D^{\alpha} y(t)=f(t, y(t)) \text {, for a.e. } t \in J=[1, T], \quad 0<\alpha \leq 1, \\
a y(1)+b y(T)=c,
\end{gathered}
$$

where ${ }_{H}^{c} D^{\alpha}$ is the Caputo-Hadamard fractional derivative, $f:[1, T] \times \mathbb{R} \rightarrow \mathbb{R}$ is a given function, and $a, b$ and $c$ are real constants such that $a+b \neq 0$.

This paper is organized as follows. In Section 2, we recall briefly some basic definitions and preliminary facts which will be used throughout subsequent sections. In Section 3, we shall provide sufficient conditions ensuring the existence of solutions for the problem (1.1)-(1.2) via applications of classical fixed point theorems. Finally in Section 4, we give examples to illustrate the theory presented in the previous sections.

\section{Preliminaries}

In this section, we introduce notations, definitions, and preliminary facts that will be used in the remainder of this paper.

Let $C([1, T], \mathbb{R})$ be the Banach space of all continuous functions from $[1, T]$ into $\mathbb{R}$ with the norm

$$
\|y\|_{\infty}=\sup \{|y(t)|: 1 \leq t \leq T\} .
$$

Let the space

$$
A C_{\delta}^{n}([a, b], \mathbb{R})=\left\{h:[a, b] \rightarrow \mathbb{R}: \delta^{n-1} h(t) \in A C([a, b], \mathbb{R})\right\},
$$

where $\delta=t \frac{d}{d t}$ is the Hadamard derivative and $A C([a, b], \mathbb{R})$ is the space of absolutely continuous functions on $[a, b]$.

Definition 2.1. ([22]). The Hadamard fractional integral of order $r$ for a function $h:[1,+\infty) \rightarrow \mathbb{R}$ is defined as

$$
{ }_{H} I^{r} h(t)=\frac{1}{\Gamma(r)} \int_{1}^{t}\left(\log \frac{t}{s}\right)^{r-1} \frac{h(s)}{s} d s, r>0,
$$

where $\Gamma$ is the Gamma function. 
Definition 2.2. ([22]). For a function $h$ given on the interval $[1,+\infty)$, and $n-1<r<n$, the Hadamard derivative of order $r$ is defined by

$$
\begin{aligned}
\left({ }_{H} D^{r} h\right)(t) & =\frac{1}{\Gamma(n-r)}\left(\frac{d}{d t}\right)^{n} \int_{1}^{t}\left(\log \frac{t}{s}\right)^{n-r-1} \frac{h(s)}{s} d s \\
& =\delta^{n}\left(H I^{n-r} h\right)(t),
\end{aligned}
$$

where $n=[r]+1$ and $[r]$ denotes the integer part of $r$, and $\log (\cdot)=\log _{e}(\cdot)$.

Definition 2.3. ([20])Let $r \geq 0$ and $n-1<r<n$, where $n=[r]+1$, and $h \in A C_{\delta}^{n}[1,+\infty)$. The Caputo-Hadamard fractional derivative of order $r$ is defined by

$$
\begin{aligned}
\left({ }_{H}^{c} D^{r} h\right)(t) & =\frac{1}{\Gamma(n-r)} \int_{1}^{t}\left(\log \frac{t}{s}\right)^{n-r-1} \delta^{n} h(s) \frac{d s}{s} \\
& ={ }_{H} I^{n-r}\left(\delta^{n} h\right)(t) .
\end{aligned}
$$

Lemma 2.4. ([20]). Let $h \in A C_{\delta}^{n}[1,+\infty)$ and $r>0$. Then

$$
{ }_{H} I^{r}\left({ }_{H}^{c} D^{r} h\right)(t)=h(t)-\sum_{i=0}^{n-1} \frac{\delta^{i} y(1)}{i !}(\log t)^{i} .
$$

The following result will be used in Subsection 3.3.

Theorem 2.5. (Nonlinear alternative for single-valued maps) [18] Let $E$ be a Banach space, $C$ be a closed, convex subset of $E, U$ be an open subset of $C$ and $0 \in U$. Suppose that $N: U \rightarrow C$ is a continuous, compact map (that is, $N(U)$ is a relatively compact subset of $C$ ). Then

(i) either $N$ has a fixed point in $U$, or

(ii) there exist $x \in \partial U$ (the boundary of $U$ in $C$ ) and $\lambda \in(0,1)$ for which $x=\lambda N(x)$.

\section{Main Results}

Let us start by defining what we meant by a solution of the problem 1.1$)-(1.2)$.

Definition 3.1. A function $y \in A C_{\delta}^{1}([1, T], \mathbb{R})$ is said to be a solution of (1.1)-(1.2) if $y$ satisfies the equation ${ }_{H}^{c} D^{\alpha} y(t)=f(t, y(t))$ on $J$, and the conditions 1.2).

For the existence of solutions for the problem (1.1)-(1.2), we need the following auxiliary lemma.

Lemma 3.2. Let $h:[1,+\infty) \rightarrow \mathbb{R}$ be a continuous function. A function $y$ is a solution of the fractional integral equation

$$
y(t)=\frac{1}{\Gamma(\alpha)} \int_{1}^{t}\left(\log \frac{t}{s}\right)^{\alpha-1} h(s) \frac{d}{d s}-\frac{b}{\Gamma(\alpha)(a+b)} \int_{1}^{T}\left(\log \frac{T}{s}\right)^{\alpha-1} h(s) \frac{d}{d s}+\frac{c}{(a+b)}
$$

if and only if $y$ is a solution of the fractional boundary value problem,

$$
\begin{gathered}
{ }_{H}^{c} D^{\alpha} y(t)=h(t), \quad 0<\alpha<1 \\
a y(1)+b y(T)=c .
\end{gathered}
$$


Proof. Assume $y$ satisfies 3.2 . Then Lemma 2.4 implies that

$$
y(t)={ }_{H} I^{\alpha} f(t)+c_{1} .
$$

The boundary condition 3.3 implies that

$$
a y(1)+b y(T)=c_{H} I^{\alpha} f(T)+(a+b) y(1)=c,
$$

so

$$
c_{1}=\frac{c-b_{H} I^{\alpha} f(T)}{(a+b)} .
$$

Finally, we obtain the solution (3.1)

$$
y(t)={ }_{H} I^{\alpha} f(t)-\frac{b}{(a+b)} H I^{\alpha} f(T)+\frac{c}{(a+b)} .
$$

Inversely, it is clear that if $y$ satisfies equation (3.1), then equations $(3.2)-(3.3)$ hold.

In the following subsections we prove existence, as well as existence and uniqueness results, for the boundary value problem (1.1)-1.2 by using a variety of fixed point theorems.

\subsection{Existence and uniqueness result via Banach's fixed point theorem}

Theorem 3.3. Assume the following hypothesis:

(H1) There exists a constant $k>0$ such that

$$
|f(t, x)-f(t, y)| \leq k|x-y| \text { for a.e. } t \in J \text { and each } x, y \in \mathbb{R} .
$$

If

$$
\left[1+\frac{|b|}{|a+b|}\right] \frac{k(\log T)^{\alpha}}{\Gamma(\alpha+1)}<1,
$$

then the boundary value problem (1.1)-(1.2) has a unique solution on $[1, T]$,.

Proof. Transform the problem (1.1)-1.2 into a fixed point problem for the operator $N$ defined by

$$
\begin{aligned}
N(y(t)) & =\frac{1}{\Gamma(\alpha)} \int_{1}^{t}\left(\log \frac{t}{s}\right)^{\alpha-1} f(s, y(s)) \frac{d}{d s} \\
& -\frac{b}{(a+b) \Gamma(\alpha)} \int_{1}^{T}\left(\log \frac{T}{s}\right)^{\alpha-1} f(s, y(s)) \frac{d}{d s}+\frac{c}{(a+b)} .
\end{aligned}
$$

Applying the Banach contraction mapping principle, we shall show that $N$ is a contraction.

Let $x, y \in A C_{\delta}^{1}([1, T], \mathbb{R})$. Then for each $t \in J$ we have

$$
\begin{aligned}
|(N x)(t)-(N y)(t)| & \leq \frac{1}{\Gamma(\alpha)} \int_{1}^{t}\left(\log \frac{t}{s}\right)^{\alpha-1}|f(s, x(s))-f(s, y(s))| \frac{d}{d s} \\
& +\frac{|b|}{\Gamma(\alpha)|a+b|} \int_{1}^{T}\left(\log \frac{T}{s}\right)^{\alpha-1}|f(s, x(s))-f(s, y(s))| \frac{d}{d s} \\
& \leq \frac{1}{\Gamma(\alpha)} \int_{1}^{t}\left(\log \frac{t}{s}\right)^{\alpha-1}|f(s, x(s))-f(s, y(s))| \frac{d}{d s} \\
& +\frac{|b|}{\Gamma(\alpha)|a+b|} \int_{1}^{T}\left(\log \frac{T}{s}\right)^{\alpha-1}|f(s, x(s))-f(s, y(s))| \frac{d}{d s} \\
& \leq\left[1+\frac{|b|}{|a+b|} \frac{k(\log T)^{\alpha}}{\Gamma(\alpha+1)}\|x-y\|_{\infty} .\right.
\end{aligned}
$$

Thus

$$
\|N(x)-N(y)\|_{\infty} \leq\left[1+\frac{|b|}{|a+b|}\right] \frac{k(\log T)^{\alpha}}{\Gamma(\alpha+1)}\|x-y\|_{\infty} .
$$

We deduce that $N$ has a unique fixed point which is the unique solution of the problem (1.1)-(1.2). 


\subsection{Existence result via Schaefer's fixed point theorem}

Theorem 3.4. Assume the hypotheses:

(H2) The function $f:[1, T] \times \mathbb{R} \rightarrow \mathbb{R}$ is continuous,

(H3) There exists a constant $M>0$ such that

$$
|f(t, u)| \leq M \text { for a.e. } t \in J \text { and each } u \in \mathbb{R} .
$$

Then the boundary value problem (1.1)-(1.2) has at least one solution on $[1, T]$.

Proof. We shall use Schaefer's fixed point theorem to prove that $N$ defined by (3.5) has a fixed point. The proof will be given in several steps.

Step 1: $N$ is continuous.

Let $\left\{y_{n}\right\}$ be a sequence such that $y_{n} \rightarrow y$ in $A C_{\delta}^{1}(J, \mathbb{R})$. Then, for each $t \in J$,

$$
\begin{aligned}
\left|\left(N y_{n}\right)(t)-(N y)(t)\right| & \leq \frac{1}{\Gamma(\alpha)} \int_{1}^{t}\left(\log \frac{t}{s}\right)^{\alpha-1}\left|f\left(s, y_{n}(s)\right)-f(s, y(s))\right| \frac{d}{d s} \\
& +\frac{|b|}{\Gamma(\alpha)|a+b|} \int_{1}^{T}\left(\log \frac{T}{s}\right)^{\alpha-1}\left|f\left(s, y_{n}(s)\right)-f(s, y(s))\right| \frac{d}{d s} \\
& \leq\left[1+\frac{|b|}{\Gamma(\alpha)|a+b|}\right] \frac{(\log T)^{\alpha}}{\Gamma(\alpha+1)}\left\|f\left(\cdot, y_{n}(\cdot)\right)-f(\cdot, y(\cdot))\right\|_{\infty} .
\end{aligned}
$$

Since $f$ is continuous, we have $\left\|N\left(y_{n}\right)-N(y)\right\|_{\infty} \rightarrow 0$ as $n \rightarrow \infty$.

Step 2: $N$ maps bounded sets into bounded sets in $C([1, T], \mathbb{R})$.

Indeed, it is enough to show that for any $\mu^{*}>0$, there exists a positive constant $l$ such that for each $y \in B_{\mu^{*}}:=\left\{y \in C([1, T], \mathbb{R}):\|y\|_{\infty} \leq \mu^{*}\right\}$, we have $\|N(y)\|_{\infty}<l$. In fact, we have

$$
\begin{aligned}
|N(y)(t)| & \leq \frac{1}{\Gamma(\alpha)} \int_{1}^{t}\left(\log \frac{t}{s}\right)^{\alpha-1}|f(s, y(s))| \frac{d}{d s} \\
& +\frac{|b|}{\Gamma(\alpha)|a+b|} \int_{1}^{T}\left(\log \frac{T}{s}\right)^{\alpha-1}|f(s, y(s))| \frac{d}{d s}+\frac{|c|}{|a+b|} .
\end{aligned}
$$

Thus

$$
\|N(y)\|_{\infty} \leq \frac{M(\log T)^{\alpha}}{\Gamma(\alpha+1)}\left[1+\frac{|b|}{|a+b|}\right]+\frac{|c|}{|a+b|}:=l
$$

Step 3: $N$ maps bounded sets into equicontinuous sets of $C([1, T], \mathbb{R})$.

Let $t_{1}, t_{2} \in J, t_{1}<t_{2}, B_{\mu^{*}}$ be a bounded set of $C([1, T], \mathbb{R})$ as in Step 2, and let $y \in B_{\mu^{*}}$. Then

$$
\begin{aligned}
\left|(N y)\left(t_{2}\right)-(N y)\left(t_{1}\right)\right| & \leq \frac{1}{\Gamma(\alpha)} \int_{1}^{t_{1}}\left[\left(\log \frac{t_{2}}{s}\right)^{\alpha-1}-\left(\log \frac{t_{1}}{s}\right)^{\alpha-1}\right]|f(s, y(s))| \frac{d s}{s} \\
& +\frac{1}{\Gamma(\alpha)} \int_{t_{1}}^{t_{2}}\left(\log \frac{t_{2}}{s}\right)^{\alpha-1}|f(s, y(s))| \frac{d s}{s} \\
& \leq \frac{M}{\Gamma(\alpha+1)}\left[\left(\log t_{2}\right)^{\alpha}-\left(\log t_{1}\right)^{\alpha}\right] .
\end{aligned}
$$

As $t_{1} \longrightarrow t_{2}$, the right-hand side of the above inequality tends to zero. As consequence of Step1 to Step 3, together with the Arzela-Ascoli theorem, we can conclude that $N$ is continuous and completely continuous.

Step 4: Apriori bounds.

Now it remains to show that the set

$$
\epsilon:=\{y \in C(J, \mathbb{R}): y=\mu N(y) \text { for some } 0<\mu<1\}
$$

is bounded. For such a $y \in \epsilon$,

$$
\begin{aligned}
y(t) & =\mu\left[\frac{1}{\Gamma(\alpha)} \int_{1}^{t}\left(\log \frac{t}{s}\right)^{\alpha-1} f(s, y(s)) \frac{d}{d s}-\frac{b}{\Gamma(\alpha)(a+b)} \int_{1}^{T}\left(\log \frac{T}{s}\right)^{\alpha-1} f(s, y(s)) \frac{d}{d s}\right. \\
& \left.+\frac{c}{(a+b)}\right] .
\end{aligned}
$$


For $\mu \in[0,1]$, let $y$ be such that for each $t \in[1, T]$

$$
\begin{aligned}
|(N y)(t)| & \leq \frac{1}{\Gamma(\alpha)} \int_{1}^{t}\left(\log \frac{t}{s}\right)^{\alpha-1}|f(s, y(s))| \frac{d}{d s} \\
& +\frac{|b|}{\Gamma(\alpha)|a+b|} \int_{1}^{T}\left(\log \frac{T}{s}\right)^{\alpha-1}|f(s, y(s))| \frac{d}{d s} \\
& +\frac{|c|}{|a+b|} \\
& \leq M \frac{(\log T)^{\alpha}}{\Gamma(\alpha+1)}\left[1+\frac{|b|}{|a+b|}\right]+\frac{|c|}{|a+b|} \\
& :=R .
\end{aligned}
$$

Thus

$$
\|N(y)\|_{\infty} \leq R
$$

This implies that the set $\epsilon$ is bounded. As a consequence of Schaefer's fixed point theorem, we deduce that $\mathrm{N}$ has a fixed point which is a solution of the problem(1)-(2)

\subsection{Existence result via the Leray-Schauder nonlinear alternative}

Theorem 3.5. Assume the following hypotheses:

(H4) There exist $\phi_{f} \in L^{1}\left(J, \mathbb{R}_{+}\right)$and $\psi:[0, \infty) \longrightarrow(0, \infty)$ continuous and nondecreasing such that

$$
|f(t, u)| \leq \phi_{f}(t) \psi(|u|) \text { for a.e. } t \in J \text { and each } u \in \mathbb{R} \text {. }
$$

(H5) there exists a number $M^{*}>0$ such that

$$
\frac{M^{*}}{\left[1+\frac{|b|}{|a+b|}\right] \psi\left(M^{*}\right)_{H} I^{\alpha} \phi_{f}(T)+\frac{|c|}{|a+b|}}>1 .
$$

Then the boundary value problem (1.1)-(1.2) has at least one solution on $[1, T]$.

Proof. We shall use the Leray-Schauder theorem to prove that $N$ defined by (3.5) has a fixed point. As shown in Theorem 3.4, we see that the operator $N$ is continuous, uniformly bounded, and maps bounded sets into equicontinuous sets. So by the Arzela-Ascoli theorem $N$ is completely continuous.

Let $y$ be such that for each $t \in[1, T]$

$$
\begin{aligned}
|y(t)| & \leq \frac{1}{\Gamma(\alpha)} \int_{1}^{t}\left(\log \frac{t}{s}\right)^{\alpha-1} \phi_{f}(s) \psi(|y|) \frac{d s}{s} \\
& +\frac{|b|}{\Gamma(\alpha)(|a+b|)} \int_{1}^{T}\left(\log \frac{T}{s}\right)^{\alpha-1} \phi_{f}(s) \psi(|y|) \frac{d}{d s}+\frac{|c|}{|a+b|} \\
& \leq\left[1+\frac{|b|}{|a+b|}\right] \psi\left(\|y\|_{\infty}\right) I^{\alpha} \phi_{f}(T)+\frac{|c|}{|a+b|} .
\end{aligned}
$$

Thus

$$
\frac{\|y\|_{\infty}}{\left[1+\frac{|b|}{|a+b|}\right] \psi\left(\|y\|_{\infty}\right) I^{\alpha} \phi_{f}(T)+\frac{|c|}{|a+b|}} \leq 1 .
$$

Then by condition (H5), there exists $M^{*}$ such that $\|y\|_{\infty} \neq M^{*}$.

Let $B_{M^{*}}:=\left\{y \in C([1, T], \mathbb{R}):\|y\|_{\infty}<M^{*}\right\}$. The operator $N$ is completely continuous. From the choice of $B_{M^{*}}$ there is no $y \in \partial B_{M^{*}}$ such that $y=\mu N(y)$, for some $\mu \in(0,1)$. As consequence of the nonlinear alternative of Leray-Schauder type, we deduce that $N$ has a fixed point $y \in B_{M^{*}}$, which is a solution of the (1.1)- (1.2).

This completes the proof. 


\section{Examples}

In this section, we present some examples to illustrate our results of the previous section.

\subsection{Example 1}

We consider the fractional boundary value problem,

$$
\begin{gathered}
{ }_{H}^{c} D^{\frac{1}{2}} y(t)=\frac{\cos ^{2} t}{\left(e^{-t}+3\right)^{2}|y(t)|}, \text { for a.e. }(t, y) \in\left([1, e], \mathbb{R}_{+}\right), \\
y(1)+y(e)=0,
\end{gathered}
$$

where $\alpha=\frac{1}{2}, T=e, a=b=1, c=0$, and $f(t, y):=\frac{\cos ^{2} t}{\left(e^{-t}+3\right)^{2}|y|}$.

We have

$$
|f(t, y)|=\left|\frac{\cos ^{2} t}{\left(e^{-t}+3\right)^{2}|y|}\right| \leq \frac{1}{9} .
$$

Choosing $M=\frac{1}{9}$, then by Theorem 3.4 , the problem $4.1-4.2$ has a solution on $[1, e]$.

\subsection{Example 2}

We consider the fractional boundary value problem,

$$
\begin{gathered}
{ }_{H}^{c} D^{\frac{3}{2}} y(t)=(\log t)^{4} \frac{y^{2} e^{-y}}{2|y|\left(e^{-y}+2\right)^{2}}, \text { for a.e. }(t, y) \in\left([1, e], \mathbb{R}_{+}\right), \\
y(1)+2 y(e)=-1,
\end{gathered}
$$

where $\alpha=\frac{1}{2}, T=e, a=1, b=2, c=-1$, and

$$
f(t, y)=(\log t)^{4} \frac{y^{2} e^{-y}}{2|y|\left(e^{-y}+2\right)^{2}}
$$

Then,

$$
|f(t, y)|=\left|(\log t)^{4} \frac{y^{2} e^{-y}}{2|y|\left(e^{-y}+2\right)^{2}}\right| \leq(\log t)^{4} \frac{|y|}{8} .
$$

Choosing $\psi(|y|)=\frac{|y|}{8}, \phi_{f}(t)=(\log t)^{4}$, and ${ }_{H} I^{\frac{1}{2}} \phi_{f}(e)=\frac{256}{63 \sqrt{\pi}}$, if

$$
\begin{gathered}
\frac{M^{*}}{\left[1+\frac{|b|}{|a+b|}\right] \psi\left(M^{*}\right){ }_{H} I^{\alpha} \phi_{f}(T)+\frac{|c|}{|a+b|}} \\
=\frac{M^{*}}{\frac{5}{24} M^{*} \frac{256}{63 \sqrt{\pi}}+\frac{1}{3}} \\
=\frac{M^{*}}{M^{*} \frac{1280}{1512 \sqrt{\pi}}+\frac{1}{3}}>1,
\end{gathered}
$$

then we can find $M^{*} \approx 0.638$ so that all the conditions of the Theorem 3.5 are satisfied. And so there exists at least one solution of problem 4.3)-4.4 on $[1, e]$. 


\section{References}

[1] R. P Agarwal, M. Benchohra and S. Hamani, A survey on existence results for boundary value problems for nonlinear fractional differential equations and inclusions, Acta Applicandae Math. 109, No. 3 (2010), 973-1033.

[2] R. P. Agarwal, M. Meehan and D. O'Regan, Fixed point theory and applications, Cambridge Tracts in Mathematics 141 Cambridge University Press, Cambridge, UK, (2001).

[3] M. Benchohra, J. R. Graef and S. Hamani, Existence results for boundary value problems of nonlinear fractional differential equations with integral conditions, Appl. Anal. 87, No. 7 (2008), 851-863.

[4] M. Benchohra and S. Hamani, Boundary value problems for differential equations with fractional order and nonlocal conditions, Nonlinear Anal. 71 (2009), 2391-2396.

[5] M. Benchohra, S. Hamani and S. K. Ntouyas, Boundary value problems for differential equations with fractional order, Surv. Math. Appl. 3 (2008), 1-12.

[6] W. Benhamida, J. R. Graef, and S. Hamani, Boundary value problems for fractional differential equations with integral and anti-periodic conditions in a Banach space, Prog. Frac. Differ. Appl. 4, No. 2 (2018), 1-7.

[7] W. Benhamida, J. R. Graef and S. Hamani, Boundary value problems for Hadamard fractional differential equations with nonlocal multi-point boundary conditions, (to appear).

[8] W. Benhamida, S. Hamani, and J. Henderson, A boundary value problem for fractional differential equations with Hadamard derivative and nonlocal conditions, PanAmerican Math. J. 26 (2016), 1-11.

[9] P. L. Butzer, A. A. Kilbas and J. J. Trujillo, Composition of Hadamard-type fractional integration operators and the semigroup property, J. Math. Anal. Appl. 269 (2002), 387-400.

[10] P. L. Butzer, A. A. Kilbas and J. J. Trujillo, Fractional calculus in the Mellin setting and Hadamard-type fractional integrals, J. Math. Anal. Appl. 269 (2002), 1-27.

[11] P. L. Butzer, A. A. Kilbas and J. J. Trujillo, Mellin transform analysis and integration by parts for Hadamard-type fractional integrals, J. Math. Anal. Appl. 270 (2002), 1-15.

[12] Z. Cui, P. Yu and Z. Mao, Existence of solutions for nonlocal boundary value problems of nonlinear fractional differential equations, Adv. Dynam. Sys. Appl. 7 (2012), 31-40.

[13] D. Delbosco and L. Rodino, Existence and uniqueness for a nonlinear fractional differential equation, J. Math. Anal. Appl. 204 (1996), 609-625.

[14] K. Diethelm and A. D. Freed, On the solution of nonlinear fractional order differential equations used in the modeling of viscoplasticity, Scientifice Computing in Chemical Engineering II. Computational Fluid Dynamics, Reaction Engineering and Molecular Properties (F. Keil, W. Mackens, H. Voss and J. Werther, eds.), Springer-Verlag, Heidelberg, 1999, pp. $217-224$.

[15] K. Diethelm and N. J. Ford Analysis of fractional differential equations, J. Math. Anal. Appl. 265 (2002), $229-248$.

[16] K. Diethelm and G. Walz, Numerical solution of fractional order differential equations by extrapolation, Numer. Algorithms 16 (1997), 231-253.

[17] A. M. A. EL-Sayed and E. O. Bin-Taher, Positive solutions for a nonlocal multi-point boundary-value problem of fractional and second order, Electron. J. Differential Equations, Number 64, (2013), 1-8.

[18] A. Granas and J. Dugundji, Fixed Point Theory, Springer, New York, 2003.

[19] J. Hadamard, Essai sur l'etude des fonctions donnees par leur development de Taylor, J. Mat. Pure Appl. Ser. 8 (1892), 101-186.

[20] F. Jarad, D. Baleanu and T. Abdeljawad, Caputo-type modification of the Hadamard fractional derivatives, Adv. Differ. Equ. 2012, No.1 (2012),1-8.

[21] A. A. Kilbas and S. A. Marzan, Nonlinear differential equations with the Caputo fractional derivative in the space of continuously differentiable functions, Differential Equations 41 (2005), 84-89.

[22] A. A. Kilbas, H. M. Srivastava and J. J. Trujillo, Theory and Applications of Fractional Differential Equations. NorthHolland Mathematics Studies, 204, Elsevier Science B.V., Amsterdam, 2006.

[23] V. Lakshmikantham and S. Leela, Nonlinear Differential Equations in Abstract Spaces, International Series in Mathematics: Theory, Methods and Applications, 2, Pergamon Press, Oxford, UK, 1981.

[24] F. Mainardi, Fractional calculus: some basic problems in continuum and statistical mechanics, Fractals and Fractional Calculus in Continuum Mechanics (A. Carpinteri and F. Mainardi, eds.), Springer-Verlag, Wien, 1997, pp. 291-348.

[25] K. S. Miller and B. Ross, An Introduction to the Fractional Calculus and Differential Equations, John Wiley, New York, 1993.

[26] I. Podlubny, I. Petráš, B. M. Vinagre, P. O’Leary and L. Dorcak, Analogue realizations of fractional-order controllers. Fractional order calculus and its applications, Nonlinear Dynam. 29 (2002), 281-296.

[27] S. G. Samko, A. A. Kilbas and O. I. Marichev, Fractional Integrals and Derivatives: Theory and Applications, Gordon and Breach, Yverdon, 1993.

[28] P. Thiramanus, S. K. Ntouyas and J. Tariboon, Existence and uniqueness results for Hadamard- type fractional differential equations with nonlocal fractional integral boundary conditions, Abstr. Appl. Anal. (2014), Art. ID 902054,9 pp. 